\title{
ILG 1: A New Integrase-like Gene that is a Marker of Bacterial Contamination by the Laboratory Escherichia coli Strain TOP 10F'
}

\author{
Wenzhi Tian, ${ }^{1}$ Kevin Chua, ${ }^{2}$ Warren Strober, ${ }^{2}$ and Charles C. Chu, ${ }^{1}$ \\ ${ }^{1}$ Department of Medicine, New York University School of Medicine, and Department of Medicine, \\ Division of Rheumatology and Allergy-Clinical Immunology, North Shore University Hospital, \\ and the North Shore-LIJ Research Institute, Manhasset, NY, USA \\ ${ }^{2}$ Mucosal Immunity Section, Laboratory of Clinical Investigation, National Institute of Allergy \\ and Infectious Diseases, National Institutes of Health, Bethesda, MD, USA
}

Accepted June 26, 2002

\begin{abstract}
Background: Identification of differentially expressed genes between normal and diseased states is an area of intense current medical research that can lead to the discovery of new therapeutic targets. However, isolation of differentially expressed genes by subtraction often suffers from unreported contamination of the resulting subtraction library with clones containing DNA sequences not from the original RNA samples.

Materials and Methods: Subtraction using cDNA representational difference analysis (RDA) was performed on human B cells from normal or common variable immunodeficiency patients. The material remaining after the subtraction was cloned and individual clones were sequenced. The sequence of one clone with similarity to integrases (ILG1, integrase-like gene-1) was used to obtain the full length cDNA sequence and as a probe for the presence of this sequence in RNA or genomic DNA samples.

Results: After five rounds of CDNA RDA, 23.3\% of the clones from the resulting subtraction library contained Escherichia coli DNA. In addition, three clones contained the sequence of a new integrase, ILG1. The full length cDNA sequence of ILG1 exhibits prokaryotic, but not eukaryotic, features. At the DNA level, ILG1 is not similar to any known gene. At the protein level, ILG1 has 58\% similarity to integrases from the cryptic P4 bacteriophage family (S clade). The catalytic domain of ILG1 contains the
\end{abstract}

conserved features found in site-specific recombinases. The critical residues that form the catalytic active site pocket are conserved, including the highly conserved R-H-R-Y hallmark of these recombinases. Interestingly, ILG1 was not present in the original B cell populations. By probing genomic DNA, ILG1 could only be detected in the E. coli TOP10F' strain used in our laboratory for molecular cloning, but not in any of its precursor strains, including TOP10. Furthermore, bacteria cultured from the mouth of the laboratory worker who performed cDNA RDA were also positive for ILG1.

Conclusions: In the course of our studies using cDNA RDA, we have isolated and identified $I L G 1$, a likely active site-specific recombinase and new member of the bacteriophage $\mathrm{P} 4$ family of integrases. This family of integrases is implicated in the horizontal DNA transfer of pathogenic genes between bacterial species, such as those found in pathogenic strains of E. coli, Shigella, Yersinia, and Vibrio cholera. Using ILG1 as a marker of our laboratory E. coli strain TOPI0F', our evidence suggests that contaminating bacterial DNA in our subtraction experiment is due to this laboratory bacterial strain, which colonized exposed surfaces of the laboratory worker. Thus, identification of differentially expressed genes between normal and diseased states could be dramatically improved by using extra precaution to prevent bacterial contamination of samples.

\section{Introduction}

New medically important molecular targets have been identified by subtraction techniques that are able to isolate differentially expressed genes between different cell types, developmental stages or different treatments of the same cell type (1). Although not frequently reported, the resulting subtraction libraries are often contaminated with "junk" sequences, including bacteria (most often Escherichia coli), yeast, mitochondrial, viral, and vector DNA (2). Increasing reliance of subtraction techniques on the

Address correspondence and reprint requests to: Charles C. Chu, North Shore-LIJ Research Institute, 350 Community Dr., Manhasset, NY 11030. Phone: (516) 562-1085, fax: (516) 562-1683, e-mail: cchu@nshs.edu. polymerase chain reaction (PCR) to amplify small starting samples and the small amounts of material remaining after subtractive hybridization (3-6) may increase the chances of obtaining this "junk" DNA by amplification of laboratory contaminants. We have used the cDNA representational difference analysis (RDA) technique (6) to perform a subtraction between human B cells from healthy and common variable immunodeficiency (CVI) individuals. A large percentage of clones from the resulting subtraction libraries consisted of contaminating "junk" E. coli DNA. In addition, we isolated a new gene with greatest similarity to bacteriophage integrases, which we have named ILG1 (integrase-like gene-1).

Bacteriophage integrases are site-specific recombinases that are critical for the bacteriophage life 
cycle in switching between lytic and lysogenic states (7). Integrases, such as the prototypic bacteriophage $\lambda$ Integrase, are essential for the integration and excision of bacteriophage DNA from host genomic DNA. These enzymes catalyze the cutting and rejoining between two DNA duplexes at a short sitespecific sequence (8). Although catalyzing a similar DNA recombination reaction, the different bacteriophage integrases can be separated by their different DNA sequence specificities.

Integrases belong to a large family of over 100 prokaryotic and eukaryotic site-specific recombinases that includes integrases, resolvases, transposases, invertases, and excisionases (9). These proteins play an important role in biology, including the movement of viral DNA into and out of host genomes, maintenance of plasmid copy number, alteration of cell surface components, and maintenance of monomeric chromosomes (10). Recently, site-specific recombinases, particularly those of the bacteriophage P4 integrase family, have been implicated in horizontal transfer of DNA among microbes, which can promote pathogenicity to humans (11). A conserved feature of this diverse group of site-specific recombinases is comprised of the amino acid residues that form the catalytic active pocket (9). Especially notable is the conserved R-H-R-Y tetrad hallmark spread between the Box I and Box II domains of this family.

ILG1 is most similar to integrases of the bacteriophage P4 family, suggesting ILG1 is not of eukaryotic origin. The sequence of ILGI lacks characteristic eukaryotic features and contains prokaryotic features. Indeed, analysis of genomic DNA demonstrates that ILG1 comes from the Escherichia coli strain, TOP10F'. Furthermore, ILG1 is a marker of TOPIOF', since it is not found in any precursor strains of E. coli. Thus, ILG1 was employed as a marker of bacterial contamination from TOP10F'.

\section{Materials and Methods}

Oligonucleotides

Oligonucleotides were synthesized by the core facility at North Shore University Hospital (Table 1). Oligonucleotides used for cDNA RDA can make three adaptor sets: adaptor set 1 consists of annealed CCC24 and CCC25, adaptor set 2 consists of annealed CCC26 and CCC27, and adaptor set 3 consists of annealed CCC28 and CCC29. The remaining primers were used either for PCR analysis and sequencing, or for 5'/3' RACE (rapid amplification of cDNA ends) experiments.

\section{Cell Culture}

B cells were purified from peripheral blood mononuclear cells (PBMC) from CVI patients and normal control blood bank donors by Ficoll-Hypaque (Amersham Pharmacia Biotech, Piscataway, NJ) density gradient centrifugation (12) followed by
anti-CD19 monoclonal antibody coated magnetic beads (Dynal, Lake Success, NY) positive selection (13). B cells $\left(>95 \% \mathrm{CD}^{+} 0^{+}\right)$were cultured in complete RPMI 1640 supplemented with $0.005 \%$ Staphylococcus aureus Cowan I (Calbiochem, San Diego, CA), $5 \mathrm{ng} / \mathrm{ml}$ interleukin-2 (R\&D Systems, Minneapolis, MN), and $50 \mathrm{ng} / \mathrm{ml}$ interleukin-10 (PharMingen, San Diego, CA) for $48 \mathrm{~h}$ at $37^{\circ} \mathrm{C}$ and $5 \% \mathrm{CO}_{2}(14)$.

Table 1. Oligonucleotides

\begin{tabular}{|c|c|}
\hline Name & Sequence $\left(5^{\prime} \rightarrow 3^{\prime}\right)$ \\
\hline $\mathrm{CCC} 24$ & AGC ACT CTC CAG CCT CTC ACC GAG \\
\hline $\mathrm{CCC} 25$ & GAT CCT CGG TGA \\
\hline $\mathrm{CCC} 26$ & ACC GAC GTC GAC TAT CCA TGA ACG \\
\hline CCC27 & GAT CCG TTC ATG \\
\hline CCC28 & $\begin{array}{l}\text { AGG CAA CTG TGC TAT CCG } \\
\text { AGG GAG }\end{array}$ \\
\hline CCC29 & GAT CCT CCC TCG \\
\hline CCC34 & ATT AAC CCT CAC TAA AG \\
\hline $\mathrm{CCC} 35$ & AAT ACG ACT CAC TAT AG \\
\hline $\mathrm{CCC} 48$ & $\begin{array}{l}\text { GAC TCG AGT CGA CAT CGA TTT TTT } \\
\text { TTT TTT TTT TT }\end{array}$ \\
\hline M13R & GGA AAC AGC TAT GAC CAT G \\
\hline $\mathrm{CCC} 131$ & ATC CCT GGC GCT ACA GAG AAG C \\
\hline $\mathrm{CCC} 137$ & $\begin{array}{l}\text { ATC TGG CAC CAC ACC TTC TAC AAT } \\
\text { GAG CTG GG }\end{array}$ \\
\hline CCC 138 & $\begin{array}{l}\text { CGT CAT ACT CCT GCT TGC TGA TCC } \\
\text { ACA TCT GG }\end{array}$ \\
\hline $\mathrm{CCC} 155$ & GCT TGA GGC AGA CGT GTA TCC G \\
\hline CCC159 & CGA GCG AAA CAC GAT GGC AGA C \\
\hline $\mathrm{CCC} 160$ & GAT GCA GCG CGT TGA CCG TAT G \\
\hline $\mathrm{CCC} 161$ & GCA AAC GAA TCA GGA TGG AGC G \\
\hline $\mathrm{CCC} 162$ & CTG CGC CCA CCA ATT CAT CAT C \\
\hline $\mathrm{CCC} 165$ & CCC ACC GTC CAG CCT GAT G \\
\hline $\mathrm{CCC} 166$ & TAT TCG CTG CGT CCT GTT C \\
\hline $\mathrm{CCC} 169$ & AGG GGG TAG ACG CCG AAA G \\
\hline $\mathrm{CCC} 170$ & CCG TGG CGA TAT TTC ATG C \\
\hline $\mathrm{CCC} 173$ & GAG GCT GCG TAC TTT GAG G \\
\hline CCC 174 & CCC CTA GAG TTT ATG CAC C \\
\hline CCC 175 & CGA TTT CCA TGC CAC TGA C \\
\hline CCC176 & TAC TTT TCT TAC GTC GCA G \\
\hline CCC403 & GGG GGA TGT AGA AAC TCA A \\
\hline CCC404 & GGA GAA CGT CAG GAG AGG C \\
\hline CCC417 & AAC AAG CGA CAG AGC GTG C \\
\hline CCC418 & GGT GTA GAG CAG GTC GGT G \\
\hline $\begin{array}{l}\text { Oligo d(T)- } \\
\text { anchor }\end{array}$ & $\begin{array}{l}\text { GAC CAC GCG TAT CGA TGT CGA CTT } \\
\text { TTT TTT TTT TTT TTV }\end{array}$ \\
\hline PCR anchor & GAC CAC GCG TAT CGA TGT CGA C \\
\hline
\end{tabular}




\section{RNA Preparation}

Total RNA was prepared from $5 \times 10^{6}$ pelleted cells that were resuspended and lysed in RNA STAT-60 (Tel-Test Inc., Friendswood, TX) using the protocol provided by the manufacturer. The aqueous RNA fraction was precipitated by isopropanol, washed, resuspended in diethyl-pyrocarbonate-treated $\mathrm{H}_{2} \mathrm{O}$, and quantitated by UV adsorption at $260 \mathrm{~nm}$. Poly $(\mathrm{A})^{+}$mRNA was prepared from $1 \times 10^{8}$ cells using a FastTrack mRNA Isolation Kit (InVitrogen, San Diego, CA) according to manufacturer's directions. Approximately $1 \mu \mathrm{g}$ poly $(\mathrm{A})^{+}$mRNA was purified from the Oligo (dT) Cellulose tablets.

\section{cDNA Representational Difference Analysis (RDA)}

CDNA RDA was performed as previously described (6). cDNA was prepared from $1 \mu \mathrm{g} \operatorname{poly}(\mathrm{A})^{+}$ mRNA using a RiboClone cDNA Synthesis System (Promega Corp., Madison, WI). Briefly, after conversion of mRNA into single stranded cDNA using Avian Myeloblastosis Virus reverse transcriptase and oligo $(\mathrm{dT})_{15}$ primer, second strand synthesis was performed with RNaseH and DNA polymerase I resulting in approximately $2 \mu \mathrm{g}$ double stranded cDNA between 200 to 2000 bp in length. The doublestranded cDNA products were digested with Sau3AI (New England Biolabs (NEB), Beverly, MA), ligated to adaptor set 1 , and PCR amplified using CCC24 to produce amplicon representations of healthy (tester) and CVI patient (driver) B cell RNA. The driver amplicon was biotinylated by PCR amplification with 5' biotinylated CCC24. The tester amplicon was modified by replacing adaptor set 1 ends with adaptor set 3 by digestion with Sau3AI, purification by ChromaSpin-100 columns (Clontech Laboratories, Palo Alto, CA), and ligation with adaptor set 3. For the first round of subtraction, the modified tester amplicon was hybridized overnight at $65^{\circ} \mathrm{C}$ with excess biotinylated driver amplicon (20:1 driver:tester). The hybridization mixture was depleted with streptavidin magnetic beads (Dynal) to remove driver DNA strands. CCC28 of adaptor set 3 was used to PCR amplify tester DNA hybrids from the depleted mixture. The resulting amplicon was modified for the subsequent subtraction by replacing adaptor set 3 with adaptor set 2 . The second round of hybridization and subtraction was performed as above except using a higher driver:tester ratio (100:1) and using CCC26 for PCR amplification. For subsequent rounds of subtraction, adaptor set 2 and adaptor set 3 were interchanged on the tester amplicon as above, the hybridizations were performed at increasing driver:tester ratios (200:1, 1000:1, 2000:1), and the subtraction repeated.

\section{Cloning, DNA Sequencing, and Sequence Analysis}

Products of the last round of CDNA RDA were either directly cloned into pCR2.1 using the Original TA Cloning Kit (InVitrogen) or first digested with Sau3AI and cloned into the BamHI site of pBluescriptSK+ (Stratagene, La Jolla, CA). After transformation of TOP10F' E. coli (InVitrogen) to ampicillin resistance, individual bacterial colonies were boiled and tested by PCR for inserts in the cloning plasmid (6). Inserts in pCR2.1 were PCR amplified using CCC35 and M13R primers, purified by Chromaspin-100 columns, and sequenced using the same primers. Inserts in pBluescriptSK + were analyzed similarly using CCC34 and CCC35 primers. DNA sequencing was performed on an ABI 373 DNA Sequencer using the ABI PRISM BigDye Terminator Cycle Sequencing Ready Reaction Kit with AmpliTaq DNA Polymerase, FS (PE Applied Biosystems, Foster City, CA). Sequences were assembled using AssemblyLIGN (Oxford Molecular, Campbell, CA) and analyzed by BLAST (15) using default settings provided by the National Center for Biotechnology Information (Bethesda, MD) to compare to public sequence databases or by MacVector (Oxford Molecular) on Macintosh computers (Apple, Cupertino, CA).

\section{Determination of Full Length ILGI cDNA Sequence}

From the sequence of the cDNA RDA clone (wzl\#1), we employed 5'/3' RACE to obtain the complete ILG1 cDNA sequence. Seven RACE clones were isolated that spanned the entire ILG1 CDNA (Fig. 1). 3' RACE was performed using ELONGase Enzyme Mix (Life Technologies, Rockville, MD) according to manufacturer's instructions. After first strand cDNA synthesis with CCC48, the 3' end was PCR amplified using CCC131 and CCC48. The resulting $1180 \mathrm{bp} \mathrm{PCR}$ product was cloned into pCR2.1 resulting in plasmids pTIAN1, pTIAN2, and pTIAN3. These plasmids are identical with the exception that the insert in pTIAN1 is in the opposite orientation. The inserts in pTIAN1-3 were sequenced with CCC35, CCC155, CCC159, CCC160, CCC161, CCC162, and M13R primers. 5' RACE was performed using a 5'/3' RACE Kit (Boehringer Mannheim, Indianapolis, IN). 5' RACE clone pTIAN7 was obtained after first strand cDNA synthesis with CCC160 primer, poly(A) tailing

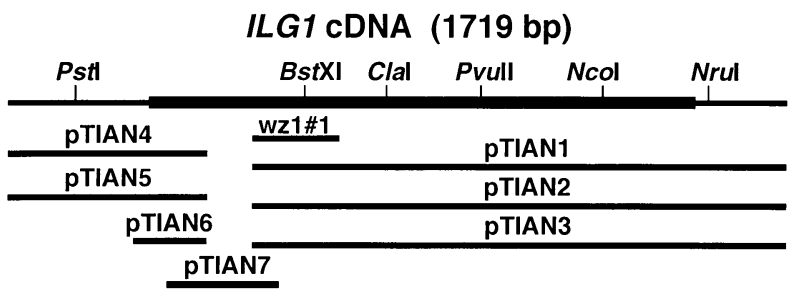

Fig. 1. ILG1 cDNA clone map. The top line diagrams the full length $I L G 1$ cDNA with the thin lines indicating the 5' and 3' untranslated regions on the left and right, respectively. The large dark box indicates the coding sequence. The locations of selected restriction enzyme sites are indicated above the line. Underneath, the locations of the ILG1 cDNA fragments contained in the original cDNA RDA clone (wzl\#l) and the subsequent RACE clones (pTIAN1-7) are indicated by dark lines. Diagram is shown to scale. 
with terminal transferase, PCR amplification with CCC165 and oligo d(T)-anchor primers, followed by a second PCR amplification with CCC166 and PCR anchor primers. A $282 \mathrm{bp}$ PCR product was inserted into pCR2.1 resulting in pTIAN7. pTIAN7 was sequenced with oligo $d(T)$-anchor and M13R primers. 5' RACE clones pTIAN4, pTIAN5, and pTIAN6 were obtained after first strand CDNA synthesis with CCCl66 primer, poly(A) tailing, PCR amplification with CCC169 and oligo $d(T)$-anchor primers, followed by a second PCR amplification with CCC170 and PCR anchor primers. A $470 \mathrm{bp}, 473 \mathrm{bp}$, or $199 \mathrm{bp}$ PCR product was inserted into pCR2.1 resulting in pTIAN4, pTIAN5, and pTIAN6, respectively. pTIAN4 and 5 were sequenced with oligo $d(T)$-anchor, M13R, CCCl70, CCC173, CCC174, and CCC176 primers. pTIAN6 was sequenced with CCC 35 and CCC 175 primers. Assembly of all the resulting sequences and removal of terminal poly(A) stretches resulted in the full length ILGI cDNA.

\section{Northern Blot Analysis}

After denaturation, total RNA samples $(10 \mu \mathrm{g})$ were electrophoresed in $1 \%$ agarose/formaldehyde gels, transferred to Hybond-N nylon membrane (Amersham Pharmacia Biotech), and UV-crosslinked. Membrane was prehybridized at $68^{\circ} \mathrm{C}$ for $\mathrm{l} \mathrm{h}$ in ExpressHyb Hybridization Solution (Clontech), hybridized with probe at $68^{\circ} \mathrm{C}$ for $16 \mathrm{~h}$, washed following the manufacturer's instructions, and then analyzed by PhosphorImager (Molecular Dynamics, Sunnyvale, CA). Probe DNA fragment (50 ng) was radiolabeled by random primer labeling kit (Stratagene) in the presence of $\left[\alpha{ }^{32} \mathrm{P}\right] \mathrm{dCTP}(3000 \mathrm{Ci} / \mathrm{mmol}$, Amersham Pharmacia Biotech). The ILGI probe was plasmid pTIANl. The human $\beta$-actin probe $(838 \mathrm{bp})$ was PCR amplified from cDNA using primers CCC137/CCC138. The 18S rRNA probe was PCR amplified using CCC $34 / C C C 35$ primers from clone wz2\#3 containing a 556 bp 18S rRNA cDNA RDA Sau3AI fragment inserted in pBluescriptSK+. Membrane was reprobed after being stripped by washing in boiling $0.5 \%$ SDS.

\section{Genomic DNA Preparation}

Human genomic DNA was prepared from peripheral blood mononuclear cells using a Puregene DNA isolation kit for buccal cells (Gentra Systems, Minneapolis, MN). Briefly, $20 \times 10^{6}$ cells were lysed in Cell Lysis Solution, extracted with the Protein Precipitation Solution, and precipitated with isopropanol. The resulting DNA was resuspended in TE buffer $(10 \mathrm{mM}$ tris(hydroxymethyl)aminomethane (Life Technologies), $1 \mathrm{mM}$ ethylenediaminetetraacetic acid (Sigma, St. Louis, MO), pH 8.0). Bacterial genomic DNA from E. coli strains TOP10, TOP10F' (InVitrogen), M182, MC1000, MC1060, MC1061, MG1655 (E. coli Genetic Stock Center, New Haven, CT), or bacteria cultured from the laboratory worker were prepared using CTAB (hexadecyltrimethyl ammonium bromide) (Sigma) (16). Swabs from the mouth, face and, hand were inoculated onto LB plates, incubated overnight at $37^{\circ} \mathrm{C}$, and resulting single colonies were picked for genomic DNA preparation. Briefly, bacteria were cultured in LB medium for $16-18 \mathrm{hrs}$ at $37^{\circ} \mathrm{C}$ with aeration, lysed, digested with proteinase $\mathrm{K}$ and precipitated with CTAB. High molecular weight DNA was recovered from the supernatant by isopropanol precipitation, resuspended in TE buffer, and quantitated by UV absorption at $260 \mathrm{~nm}$.

\section{Southern Blot and Dot Blot Analysis}

Genomic DNA was digested with Sau $3 \mathrm{AI}$ at $37^{\circ} \mathrm{C}$ overnight. $10 \mu \mathrm{g}$ DNA was electrophoresed in $0.7 \%$ agarose gels, transferred by Southern blot to Hybond-N, membranes and probed as above (see Northern blot analysis).

For dot blots, $1 \mu \mathrm{g}$ DNA was directly spotted onto membranes and treated as above. Like the 18S rRNA probe, the lacI probe was PCR amplified from clone wzl\#15 containing a 108 bp lacl Sau3AI fragment. The $f r d A$ probe was PCR amplified either from cDNA RDA clone wzl\# 103 (contains a 278 bp $f r d A$ fragment plus $72 \mathrm{bp}$ of adaptor inserted in the TA cloning site of pCR2.1) using M13R/CCC35 primers or from $E$. coli genomic DNA using CCC403/CCC404 primers ( $183 \mathrm{bp}$ product). The selC probe was PCR amplified from E. coli genomic DNA using CCC417/CCC418 primers to generate a $293 \mathrm{bp}$ product.

\section{Results}

Contamination of cDNA RDA Subtraction Clones

To isolate genes expressed by $\mathrm{B}$ cells during isotype class switch, subtraction by cDNA RDA was performed between healthy (tester) and CVI patient (driver) $B$ cells cultured under conditions where only the healthy B cells undergo class switching (14). After five rounds of subtraction, Southern blot analysis indicated that tester DNA fragments had been enriched (data not shown). This material was cloned and 103 randomly picked clones were sequenced. After sequence comparison, these clones could be grouped into 52 genes (many genes were represented by multiple clones) (Table 2). Of these genes, 15 could be functionally categorized based on sequence similarity to genes involved in enzyme reactions (6), signal transduction (4), transcription (2), secreted products (1), cell cycle (1), and DNA recombination (1). A surprising number of unknown genes (39 clones representing 21 genes) were isolated that did not match any gene or protein of known biological function. Finally, a large number of identified clones were not of human origin, but represented contaminating E. coli (24 clones representing 11 genes) and plasmid (10 clones representing 5 genes) DNA fragments. When this cDNA RDA experiment was repeated a second time using three rounds of subtraction with higher driver to tester amplicon ratios (100:1, 1000:1, and 10,000:1), a significant number of clones containing E. coli DNA were still obtained ( $12.9 \%$ of 93 clones). 
Table 2. Sequence identification of cDNA RDA clones

\begin{tabular}{|c|c|c|c|c|}
\hline Function & Similarity* or Name & $\begin{array}{c}\text { Genbank } \\
\text { Similarity* }\end{array}$ & $\begin{array}{l}\text { No. of } \\
\text { Clones }\end{array}$ & Freq.(\%) \\
\hline cell cycle control & HRAD9 & HSU53174 & 1 & $1.0 \%$ \\
\hline enzyme & ATP citrate lyase & HSU18197 & 8 & $7.8 \%$ \\
\hline enzyme & lysyl hydroxylase 3' splice variant & AF046889 & 2 & $1.9 \%$ \\
\hline enzyme & aldehyde reductase & HUMALR & 1 & $1.0 \%$ \\
\hline enzyme & (alanine aminotransferase) & $(461498)$ & 1 & $1.0 \%$ \\
\hline enzyme & (HMG CoA lyase) & $(14714839)$ & 1 & $1.0 \%$ \\
\hline enzyme & (maoC dehydrogenase) & $(6458985)$ & 1 & $1.0 \%$ \\
\hline product & SP-D & HUMSPD02 & 1 & $1.0 \%$ \\
\hline recombination & (integrase) "ILG1" & $(1788783)$ & 3 & $2.9 \%$ \\
\hline signal transduction & Maxp 1 & AF002251 & 3 & $2.9 \%$ \\
\hline signal transduction & MAP kinase kinase 3 & HUMMKK3A & 2 & $1.9 \%$ \\
\hline signal transduction & $\mathrm{PI}_{2}$ 5-phosphatase & HSU45973 & 2 & $1.9 \%$ \\
\hline signal transduction & casein kinase I epsilon & HUMCSNKIE & 1 & $1.0 \%$ \\
\hline transcription & TLE3 Enhancer of split & HUMTLEIII & 2 & $1.9 \%$ \\
\hline transcription & RNPS 1 & AF015608 & 1 & $1.0 \%$ \\
\hline unknown & 21 gene fragments & & 39 & $37.9 \%$ \\
\hline E. coli & 11 gene fragments & various & 24 & $23.3 \%$ \\
\hline plasmid & 5 gene fragments & various & 10 & $9.7 \%$ \\
\hline Total & & & 103 & $100.0 \%$ \\
\hline
\end{tabular}

*Similarity at only the amino acid level is indicated by parentheses.

Isolation of a New Integrase Gene, ILG1

The clones containing the DNA recombination gene obtained in our first experiment had some similarity to integrases at the amino acid level (45\% identical over 44 residues), but had no similarity at the DNA level (data not shown). Therefore, this gene was named ILG1. Using the RACE technique, we obtained the full-length cDNA sequence of ILG1 (Fig. 2). The cDNA sequence contains at least six features that are not eukaryotic, but more prokaryotic in nature. First, ILG1 has a 311 bp 5' untranslated region (UTR). Eukaryotic cDNA characteristically have a short $5^{\prime}$ UTR of 20-100 bp (17). Second, the ILGI translation start is not the first methionine (Met) codon, but the second Met codon from the $5^{\prime}$ end. Typically, the first Met codon from the $5^{\prime}$ end is the start codon in eukaryotic cDNA (18). Third, ILG1 cDNA exhibits a typical $E$. coli ribosomal binding site (5/8 bp match) that exhibits the appropriate spacing from the start codon (19). This feature allows E. coli ribosomes to start translation at internal start codons in a mRNA without having to scan the $5^{\prime}$ end and begin at the first start codon. Thus, E. coli cDNA may have long 5' UTRs with many upstream Met codons. Fourth, ILG1 does not have a typical eukaryotic Kozak sequence surrounding the start codon for optimal translation (18). The most conserved Kozak sequence features, a purine nucleotide at position -3 and a guanine nucleotide at position +4 from the first nucleotide of the start codon, are not found. Fifth, ILG1 does not have a typical eukaryotic polyadenylation site (AATAAA) to signal transcription termination (20). Instead, a possible E. coli rho-independent transcription termination site is found at the $3^{\prime}$ end, a small GC-rich stem-loop structure (21). Sixth, ILG1 codon usage is most optimal using bacterial codon usage tables (22) (data not shown).

\section{ILG1 Belongs to the Bacteriophage P4 Integrase Family}

The ILG1 coding sequence encodes a predicted 401 amino acid protein of $45 \mathrm{kDa}$. BLAST comparison of the predicted protein sequence to the public protein databases detected similarities to several integrases, with highest similarity $(\sim 58 \%)$ to the bacteriophage P4 family of integrases. Ten of these are shown in Table 3. These include those from $E$. coli bacteriophage P4 (23) and relatives (bacteriophage $\phi$-R73 (24), cryptic P4 prophages $(25,26)$ ), as well as integrases from other bacterial strains that are associated with horizontal gene transfer of large DNA segments. These DNA segments are associated with pathogenicity in humans (Shigella flexneri $(27,28)$, Yersinia pseudotuberculosis (29), 
1 CTTCTTCTTT TCTITTTTAC TTTTCTTACG TCGCAGTGAT TTCGACGGGA CGCCAATCCC GATCACGCCT CGITGGAGCG

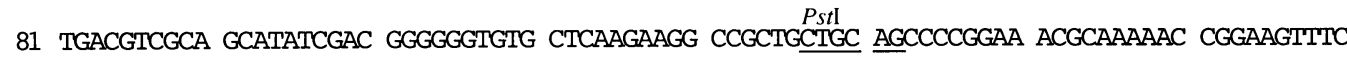
161 GAGGGAAACT TCCGGTCTIG TGTGTGGTCC CGCCGCCAGG AACCGCATTT GGCTCTGCAA GGTGCATAAA CTCTAGGGGT RBS $174{ }^{\circ}$ 241 AGACACGTCA TTATAGCGTG TACCCCCGTA TGTACCCCCA CATTGTTCCC CCC PBAAAAAC GTTCGATTTC QATGCCACTG 321 ACAGACCTCA AAGTACGCAG CCTCACAACC CCCGGTCGAT ACGCAGATGG GGGCGGCATG TACCTACAAA TTACCGCAAG 401 CGGTGGACGC TACTGGCGCA TGAAATATCG CCACGGTGGC AAGGAGAAGC TGCTCGCTTT CGGCGTCTAC CCCCTTGTGG 481 GCATCAAGGA GGCACGCGAC AAACTCTTCT ACGCGAAGCA GGCCCTCAGC CGAGGTGAAG ACCCTGGCGC TACAAAGAAG 131 561 CTCGCCAAGT TCCAAGCCGA ACAGGACGCA GCGAATACGT TCAAGGCCGT GGCGGAAGAT TGGCTTGACC ATCAGGCTGG 641 ACGGTGGGAA GCCATCACAT TGAGCCGGAC CCGAGCATCG CTTGAGGCAG ACGTGTATCC GACGCTIGGA AGCCTGCCTA 721 TATCCTCGAT CACGCCGCGC CAAGTGATGG ACGTGGTCAA GAAAATCGAG AAAAGAGGGG CAAGCGAAAT CGCCACGCGT 801 GTGCTCCAGC GGATCAAATC GATTTTTCGG TACGGTACGA CGCATCAGCG CATCGAGCGA AACACGATGG CAGACCTGGT 881 GCCCTCTGAG ATCTTGAAGC CGAGGCGCGT GCAGCACAGG GCGGCCCTGG CACCTGCTCA ACTICCAGAG TTCCTGAAAA 961 AGCTCGCGTC CTACGAAGGC GAGCCACATA CGGTCAACGC GCTGCATCTT CTAATGCTCA PVALCTGCACG CCCCGGCGAA 1041 GTGCGCGGGG CGCGCTGGGA TGAGATTGAC TTGAAAGCCA AACTCTGGCG CATCCCCCCG GAGCGGATGA AAATGCGTAG 1121 TGAACATGTG GTCCCCCTGT CCAGCGAGGC CGTAGCGGCC ATCGAGGCCA TGCGTCCGCT GAGCGGCAAC CGAGATCTGG 1201 TTTTCCCGAG CCCGTITTAT CCTTCGAAAC CGCTCAGCGA GAACACCITC AACAGCGCTA TGGCGCGGAT GGGTTACAAG 1281 TACCTCGCTA CGGCCCATGG TTTTCGGGCG CTTTTCTCCA CCGTIGCAAA CGAATCAGGA TGGAGCGCCG ACGCGATCGA 1361 ACGTCAACTT GCGCATGCGG AGCGCAATCA GGTACGCGCA GCATATGACC GGGGAGTTCG ACTCCCTGAA CGAGTGAAGA 1441 TGATGAATTG GTGGGCGCAG TATCTTGCGG AACGCAAAGC TGGCAAGGCC ATCTCGTCGC CTGAGGITCA GGAGГ⿴囗十)ACT

1521 TTCAGGTTCG NruI 1601 TAGACTACTT CTCACAGCGG ACCGCGCCTT TTTTGCGCTA ATCCCCTCCC GCTTCTGGGA GCGCCGTGCG AGCAGCGGAA 1681 CGCAAACAAC GGTTGACGCT TCTCTGTAGC GCCAGGGAT 1719

Fig. 2. ILGI cDNA sequence. The complete ILG1 cDNA sequence (Genbank accession no. AF251435) is shown in the 5' to 3' direction with nucleotide numbering of the final nucleotide and the first nucleotides in the left hand position of each line. Half arrows (labeled by numbers without the CCC prefix) underline the locations of oligonucleotides (with the arrowhead indicating the 3' end). Selected restriction enzyme sites are indicated with underlined sequence labeled above with the enzyme name. Boxed bold sequences indicate the translation start (ATG) and stop (TAA) codons. The RBS labeled boxed sequence indicates the putative bacterial ribosomal binding site, a 5/8 bp match to the ideal sequence TAAGGAGG located 5-10 nucleotides upstream of the start codon (19). A potential 3' stem loop structure involved in bacterial transcription termination is indicated by overlined inverted half arrows. Sequence obtained from the original cDNA RDA clone ranged from position 540 to 731 . Sequence from pTIAN1, 2, and 3 began at position 540 and ended at the $3^{\prime}$ end of the cDNA. Sequence from pTIAN4, 5, and 6 began at position 436 and ended at the $5^{\prime}$ end of the cDNA (pTIAN4 and 5) or at position 276 (pTIAN6). Sequence from pTIAN7 ranged from position 351 to 597.

Y. pestis (30), Vibrio cholera (31)) or colonization in plants (Mesorhizobium loti (32)). Despite similarity that extends the entire length of the protein (Fig. 3), BLAST comparison to the public nucleotide databases did not detect any DNA sequence similarities (data not shown).

\section{ILG1 Belongs to the Integrase Family}

of Site-specific Recombinases

ILG1 similarity to bacteriophage P4 family integrases includes both the $\mathrm{N}$-terminal domain that aids in DNA-binding, and the C-terminal catalytic domain (Fig. 3). The C-terminal catalytic domain has many conserved features among a diverse group of sitespecific recombinases (9). ILG1 matches this consensus sequence (59\% similar) as well as other bacteriophage P4 family integrases (Fig. 3). First, ILG1 contains the hallmark of these site-specific recombinases, a nearly invariant R-H-R-Y tetrad (9), in the appropriate location (R240, H329, R332, and Y365). Second, the $\mathrm{R}-\mathrm{H}-\mathrm{R}-\mathrm{Y}$ tetrad is found within two longer conserved regions called Box I (26 amino acids containing the first R) and Box II (4l amino acids containing H-R-Y) (9). ILG1 is fairly similar to the consensus in both Box 1 $(65 \%)$ and Box II $(48 \%)$. Third, additional smaller 
Table 3. ILG1 Belongs to the bacteriophage $\mathrm{P} 4$ family of integrases

\begin{tabular}{|c|c|c|c|c|c|}
\hline Organism & Element & Gene & $\begin{array}{c}\text { Accession } \\
\text { Number }\end{array}$ & $\begin{array}{c}\text { Identity } \\
(\%)\end{array}$ & $\begin{array}{c}\text { Similarity } \\
(\%)\end{array}$ \\
\hline E. coli TOP10F' & ${ }^{*}$ Cryptic P4 prophage $\phi$-TOP10F & $I L G 1$ & AAK49816 & 100 & 100 \\
\hline S. flexneri M90T & Shigella pathogenicity island 2 & Int & AAD44730 & 40 & 58 \\
\hline $\begin{array}{l}\text { E. coli O157:H7 } \\
\text { (EDL933) }\end{array}$ & Cryptic P4 prophage 933L & CP4 Int & AAC31482 & 41 & 58 \\
\hline S. flexneri SA100 & Shigella pathogenicity island 2 & Sai Int & AAD54663 & 40 & 58 \\
\hline M. Loti ICMP3153 & Symbiosis island & IntS & AAC24508 & 42 & 58 \\
\hline- & Bacteriophage P4 & Int & VINT_BPP4 & 41 & 57 \\
\hline E. coli K-12 MG1655 & Cryptic P4 prophage & $\operatorname{IntB}$ & INTB_ECOLI & 39 & 55 \\
\hline E. coli $\mathrm{Cl}-23$ & Bacteriophage $\phi$-R73 & Int & A42465 & 35 & 55 \\
\hline $\begin{array}{l}\text { Y. pseudotuberculosis } \\
\text { IP32637 }\end{array}$ & High-pathogenicity island & Int & CAA08754 & 36 & 55 \\
\hline Y. pestis KIM6+ & High-pathogenicity island & Int & AAC69581 & 35 & 54 \\
\hline V. cholera $\mathrm{O} 395$ & Colonization gene cluster & Int & AAC44230 & 35 & 55 \\
\hline
\end{tabular}

*Tentative proposal based on ILG1 similarity to other bacteriophage P4 integrase family members shown here.

regions of conservation called Patches I, II, and III are found in these site-specific integrases (9). ILG1 matches the three Patches fairly well $(71 \%, 57 \%$, and $100 \%$ similar, respectively).

Although prokaryotic and eukaryotic site-specific recombinases share many features, a few differences remain (9). ILG1 contains these prokaryotic features. First, in Box II, the separation between the $\mathrm{H}$ and $\mathrm{Y}$ residues of the $\mathrm{R}-\mathrm{H}-\mathrm{R}-\mathrm{Y}$ hallmark is typically 33-35 amino acids in prokaryotic recombinases, whereas eukaryotic recombinases have a 37-40 amino acid separation. ILG1 has a 35 amino acid separation between these residues (Fig. 3). Second, in Box I, the consensus prokaryotic T-G-X-R motif is replaced by $\mathrm{N}-\mathrm{C}-\mathrm{C}-\mathrm{R}$ in eukaryotic recombinases. ILG1 has a more prokaryotic-like T-A-A-R (position 237-240) at this location. Third, in Box II, the four residue prokaryotic motif L-L-G-[H,W] is replaced by a five residue motif $[\mathrm{L}, \mathrm{V}, \mathrm{S}, \mathrm{P}]-[\mathrm{Y}, \mathrm{F}, \mathrm{L}, \mathrm{V}]-\mathrm{G}-\mathrm{N}-\mathrm{W}$. ILG1 has a more prokaryotic four residue motif (Q-L-A-H, position 352-355).

\section{ILG1 is Not of Human Origin and is Present in Bacteria}

To determine if ILG1 is expressed in human cells, we examined its expression by Northern blot and found that ILG1 is not expressed in human B cells (Fig. 4). To test if $I L G 1$ is of human origin, we probed human genomic DNA by Southern blot and found that ILGI could not be detected (Fig. 5A). Because of the high percentage of contaminating $E$. coli and plasmid sequences in our cDNA RDA library (Table 2), we reasoned that ILG1 may represent a previously uncharacterized bacteriophage integrase found in the contaminating $E$. coli. Therefore, we probed genomic DNA from bacteria for the presence of ILG1 (Fig. 5A). ILG1 could easily be detected in genomic DNA from TOP10F'.
ILG1 was Recently Introduced into TOP10F'

To determine if $I L G 1$ is present in other E. coli strains, especially those that preceded TOP10F', we examined some of these strains. TOP $10 \mathrm{~F}^{\prime}$ was generated by introducing an $\mathrm{F}^{\prime}$ episome containing $\operatorname{lacl}^{q}$ and Tn10(tet ${ }^{\mathrm{R}}$ ) into strain TOP10 (InVitrogen). TOP10 comes from DH10B, which was derived from MC1061 (33). The derivation of MC1061 is complex, involving over 10 mutagenesis, transduction, and conjugation steps from the original $E$. coli $\mathrm{K}-12$ strain (Mary Berlyn, pers. com.). In brief, MC1061 was derived from MC1060 conjugated with D7091 Hfr. MC1060 was derived from MC1000 conjugated with an $\mathrm{F}^{\prime}$ strain TSM100. MC1000 was derived from a cross between M182 and D7091F. The derivation of D7091F involves at least seven more genetic manipulations from E. coli K-12. The original K-12 strain probably does not contain ILG1, because it is not found in the complete $E$. coli genomic sequence (26). This sequence was determined from E. coli strain MG1655, which is derived from the original $E$. coli $\mathrm{K}-12$ strain by two direct steps (UV treatment and growth on blood agar to $\lambda^{-}$and $r p h-1$, acridine orange treatment to $\mathrm{F}^{-}$) (34). We probed genomic DNA from MGl655 and immediate precursors of TOP10F' (Fig. 6). ILG1 can only be detected in TOP10F' and not in any precursor strains, including TOP10. Confirming our sequence comparison with the E. coli genomic sequence, MG1655 does not contain ILG1. In contrast, E. coli genes, selC and frdA, are detected in all strains.

\section{Laboratory Bacteria is Detectable on Laboratory Worker}

In order to determine the origin of bacterial contamination, we prepared genomic DNA from bacteria 

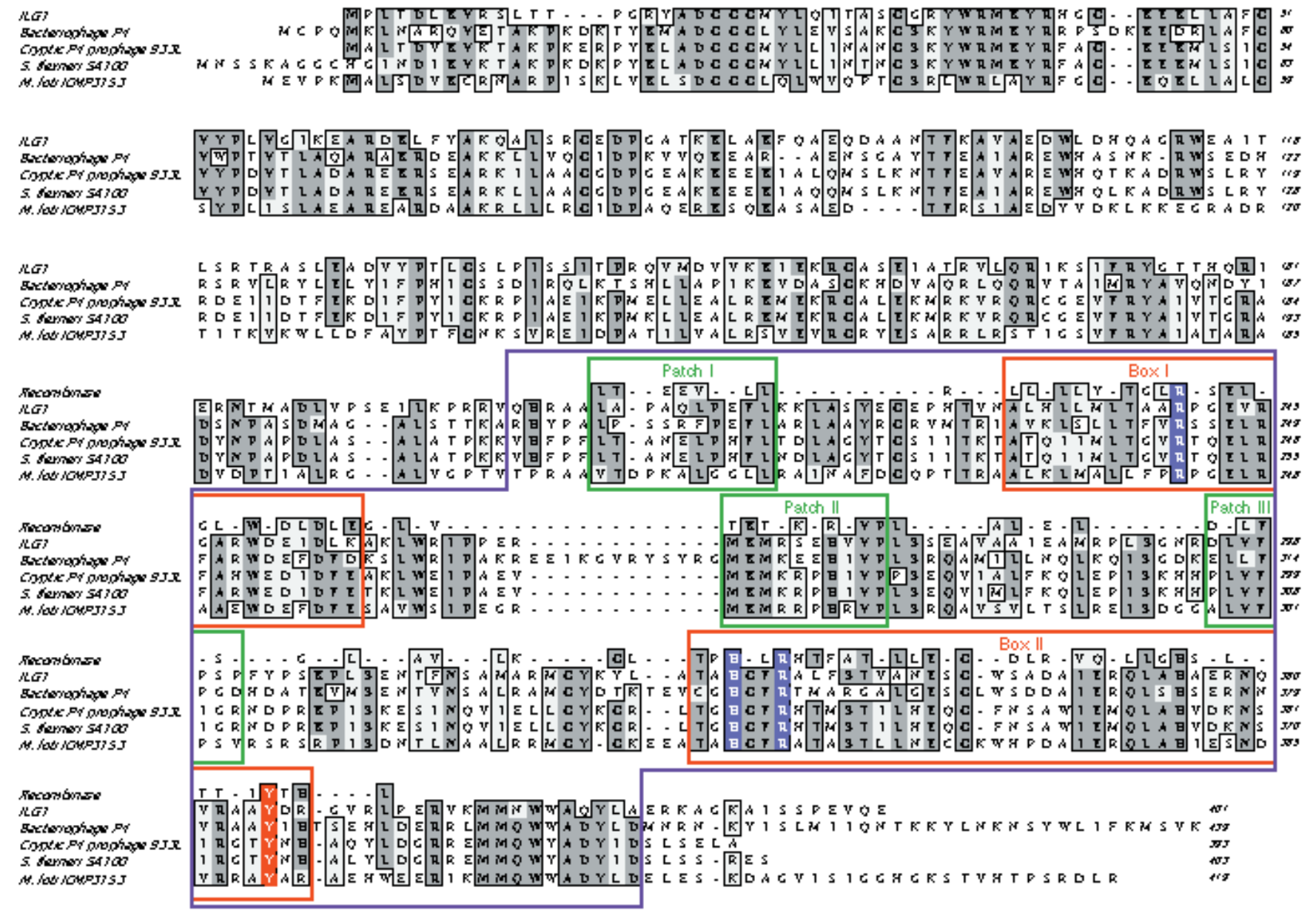

Fig. 3. ILG1 is a site-specific recombinase belonging to the bacteriophage P4 integrase family. An alignment (ClustalW) of the complete amino acid sequences of ILG1, the four most similar integrases, and the site-specific recombinase consensus catalytic domain is shown using the BLOSUM30 similarity matrix. Amino acid position of the rightmost residue of each integrase is shown for each line. Among the aligned sequences, outlined groups of amino acids indicate identical matches (shaded) and conservative substitutions $(>60 \%)$. The catalytic domain is outlined in purple. Highly conserved site-specific recombinase Box and Patch domains are boxed in red and green, respectively, with the nearly invariant R-H-R-Y hallmark shown in blue or red (active site) reverse text.

cultured from the mouth, face, and hands of the laboratory worker performing cDNA RDA. Probing these bacterial DNAs by Southern blot revealed that ILG1 was present in TOPIOF' and bacteria grown from the mouth and possibly face of the laboratory worker (Fig. 5B). We confirmed these results by dot blot, probing genomic DNA with ILG1, two E. coli sequences we obtained from the cDNA RDA subtraction ( $f r d A, l a c l)$, and 18S rRNA (Fig. 5B). ILG1, frdA, and lacI are found in TOP10F' and in bacteria cultured from the laboratory worker's mouth. 18S rRNA is found in human PBMC, but not in bacterial DNAs.

\section{Discussion}

ILG1 Belongs to the S Clade (Bacteriophage P4 Family) of Integrases

Using cDNA RDA, we have isolated ILGl, a new prokaryotic integrase-like gene with significant amino acid sequence similarity to integrases of the bacteriophage P4 family (Table 3, Fig. 3), but not to other integrase families as detected by BLAST analysis. To confirm this conclusion and detect similarities to other integrase families, ILGl was compared directly to a collection of 24 different bacteriophage integrases by phylogenetic analysis (Alvin J. Clark, pers. com.). These integrases may be grouped by similarity into four different families: the D, Q, E, and S clades (35). This phylogenetic analysis confirms that ILGl belongs to the $\mathrm{S}$ clade, containing bacteriophage P4 integrases (Alvin J. Clark, pers. com.).

Understanding this family of integrases may be of medical importance, because they often are involved in the horizontal transfer between bacteria of large segments of DNA that promote pathogenicity (11). The next most related family of integrases is the E clade, represented by bacteriophage $\lambda$ and P2 1 integrases. The $\mathrm{Q}$ clade, represented by four integrases 

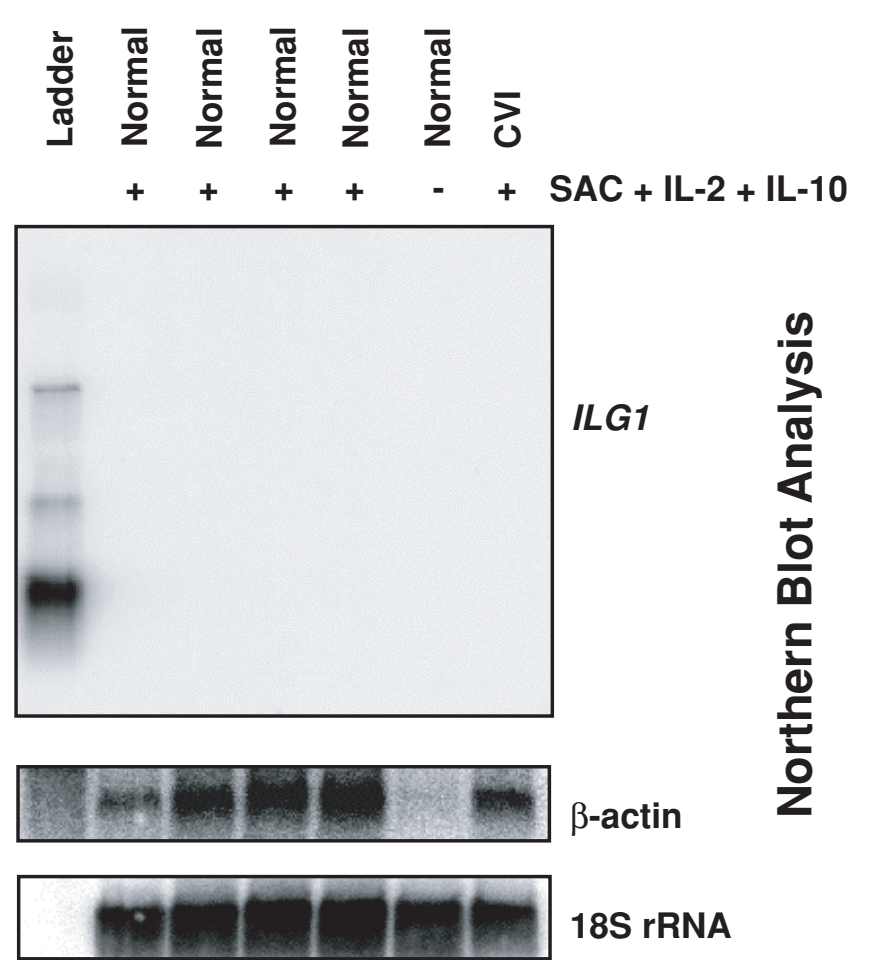

Fig. 4. ILG1 RNA is not present in stimulated human B cells. RNA samples from CVI and normal B cells cultured with SAC, IL-2, and IL-10 were Northern blotted and probed for ILG1 expression. The blot was stripped and reprobed for human $\beta$-actin and then $18 \mathrm{~S}$ rRNA expression. Ladder is $(0.24$ to $9.5 \mathrm{~kb})$ RNA ladder molecular size standards (Life Technologies).

including that from the cryptic E. coli RAC prophage, was the third most similar family. The least similar family was the D clade, represented by the bacteriophage P22 integrase.

\section{ILG1 is a Site-specific Recombinase}

Not only is ILG1 similar to bacteriophage integrases, ILG1 shares features with the integrase family of site-specific recombinases (Fig. 3). A comparison of these site-specific recombinases reveals a consensus sequence that preserves amino acid residues that generally also have a conserved position in those proteins whose crystal structures are available (9). These positions mostly comprise those in the hydrophobic core required for proper protein folding and those that comprise the catalytic active pocket. Mutational analysis supports the critical importance of the consensus amino acids for proper recombinase function. ILGI not only is similar to this recombinase consensus, which includes the box I and II, as well as patch I, II, and III regions, it exactly conserves the R-H-R-Y tetrad hallmark of this family (Fig. 3). The available crystal structures show that these critical residues form a catalytic pocket (36-39). The triad $\mathrm{R}-\mathrm{H}-\mathrm{R}$ residues form a cluster on the protein surface located at the center of interaction with the DNA substrate. The active site $\mathrm{Y}$ is found in the catalytic
A.
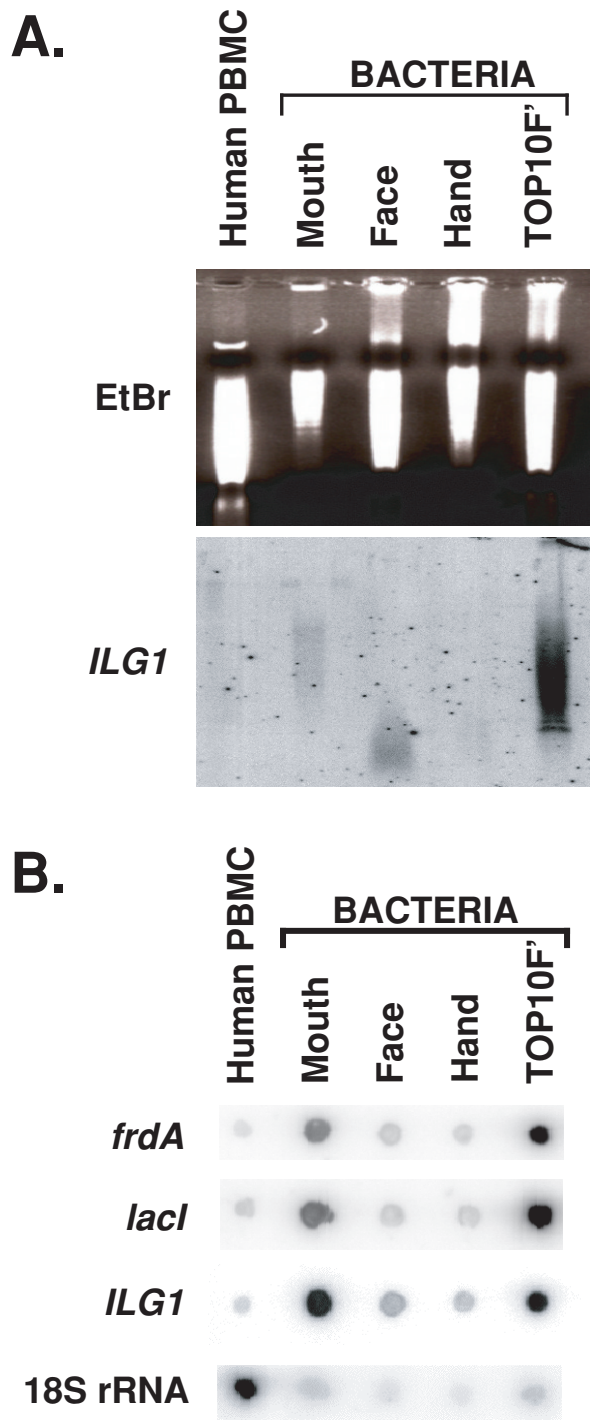

Fig. 5. ILG1 is present in bacteria cultured from lab worker, but not in human genomic DNA. (A) Digested genomic DNA from human and bacterial samples was gel electrophoresed, stained with ethidium bromide (EtBr) and photographed. The gel was Southern blotted and probed with ILG1. (B) Digested genomic human and bacterial DNA was dot blotted and probed with bacteriophage $I L G 1$. The blot was repeatedly stripped and probed with E. coli frdA, lacI, and human 18S rRNA.

pocket in a cis or trans conformation (8). In addition, ILG1 has three additional conserved residues found in the region of the active site $(9,38)$. 1) In box II, the $\mathrm{H}$ at position 355 in ILG1 is strongly conserved among this family of recombinases. Based on crystal structures, this conserved position may contribute a hydrogen bond to the stabilization of the DNA substrate in the active site. 2) The strongly conserved first $\mathrm{K}$ residue in Patch II (K267 in ILG1) contacts the DNA substrate two nucleotides adjacent to the cleavage site. 3) In Box I, the first R (position 240 in ILG1) of the R-H-R-Y hallmark forms a water bridge with an adjacent strongly conserved D 


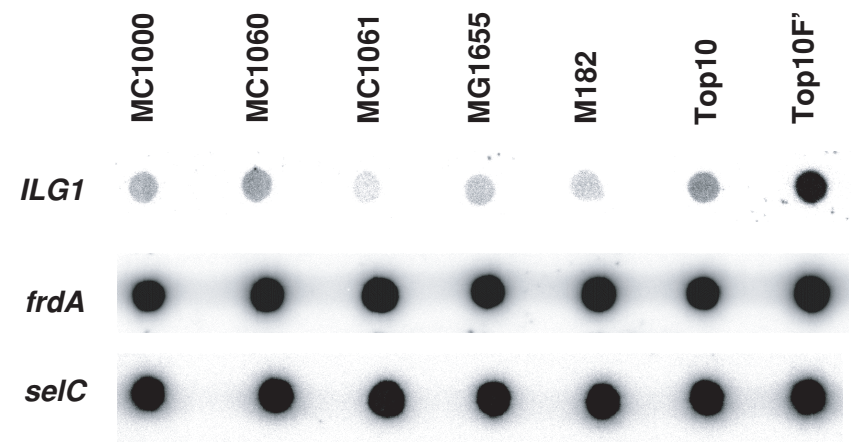

Fig. 6. ILG1 was introduced recently into TOP10F'. Genomic DNA from indicated E. coli strains were dot blotted and probed with ILG1, frdA, or selC DNA fragments.

or $\mathrm{E}$ residue (E243 in $I L G 1)$. Since ILG1 has conserved the critical residues composing the active site region, ILG1 is likely an active site-specific recombinase.

\section{Other ILG1 Sequence Features}

ILG1 not only shares the conserved site-specific recombinase features that includes Box I, II, Patch I, II, III, and the active site, but also shows some similarity in the intervening sequence between these regions (Fig. 3). In this site-specific recombinase family, the intervening sequences generally represent loops in the protein structure and show the greatest variability, including in length (9). For example, the separation between Box I and Patch II can vary from 6 to 48 amino acids. ILG1 contains 10 amino acids separating these two regions, whereas bacteriophage $\mathrm{P} 4$ integrase contains 22. In this aspect, ILG1 may be more similar to cryptic P4 prophage integrases. Despite this variation in length, the separation between all conserved Box and Patch regions in ILG1 falls within previously determined ranges for the recombinase consensus (9).

Sequence variability of these intervening sequences may serve to modify the recombinase reaction. For example, the sequence between Patch III and Box II is critical for sequence-specific recognition of the particular DNA site for cleavage. Mutations in this region of the bacteriophage $\lambda$ Integrase alter the preference for a particular DNA sequence (9). Unlike most of the intervening sequence, this particular area forms an $\alpha$-helix. Hydrophilic residues ( $\lambda$ Integrase S282, G283, R287) of this amphipathic helix form the critical DNA recognition component. ILG1 conserves hydrophobic residues that may be involved in formation of this helical structure (Fig. 3.). However, ILG1 contains different hydrophilic residues (ILG1 E310, N311, S315). Thus, ILG1 is predicted to have a different DNA recognition sequence than that of bacteriophage $\lambda$ Integrase.
Recent Introduction of ILG1 into TOP1OF'

ILG1 appears in TOP $10 \mathrm{~F}^{\prime}$ after the introduction of the $\mathrm{F}^{\prime}$ episome into TOP10 (Fig. 6). Since ILG1 is most similar to bacteriophage P4 integrase family members that are involved in horizontal DNA transfer of large DNA modules (Table 3), we tentatively propose that $I L G 1$ resides on a cryptic prophage $\phi$-TOP10F. Cryptic prophages become part of the DNA element involved in transfer of these large DNA modules between bacteria (11). In these elements, the cryptic prophage integrase gene is located next to the site of insertion, which is typically a tRNA gene. Thus, $\phi$-TOP10F may reside on the $\mathrm{F}^{\prime}$ episome or may have entered TOP10 by an independent $\phi$-TOP10F bacteriophage infection or by a $\phi$-TOP10F mediated horizontal transfer of DNA between bacteria. The origin of the $\mathrm{F}^{\prime}$ episome is unknown (InVitrogen). To preliminarily test for independent $\phi$-TOP10F insertion in the E. coli genome, we examined tRNA sites typically used by the cryptic bacteriophage P4 family. The selC tRNA gene is the site of insertion for cryptic P4 prophage 933L, bacteriophage $\phi$-R73, and Shigella pathogenicity island $2(24,25,27,28)$. The Phe and Asn tRNA genes are sites of insertion for M. loti symbiosis island and Yersinia High-pathogenicity islands, respectively $(29,32)$. The leuX tRNA gene and the $s s r A$ gene, which are sites of insertion for bacteriophage P4 and $V$. cholera colonization gene cluster, already contain prophage insertions as judged from the E. coli MG1655 genomic sequence. The F plasmid does not contain tRNA genes (Genbank Accession No. NC_002483). Therefore, we examined TOP10F' genomic DNA for insertions in the selC, pheU, pheV, asnT, asnU, asnV, and asnW by PCR across these genes. No insertions were found in these locations (data not shown). Thus, the proposed $\phi$-TOP10F bacteriophage likely resides on the introduced $\mathrm{F}^{\prime}$ episome or possibly another infrequently used tRNA insertion site.

\section{Bacterial Contamination of cDNA RDA}

Subtraction techniques often are challenged by contaminating "junk" DNA. In two separate cDNA RDA experiments, the frequency of this contamination was $35.9 \%$ and $12.9 \%$. In one of these experiments, ILG1, a unique bacteriophage integrase-like gene found only in TOP10F', was isolated. This suggests that the contamination originated from this laboratory strain of $E$. coli that we use for routine cloning. TOP10F' appears to have colonized the laboratory worker, because E. coli genes and ILGI were detected in bacteria sampled from the laboratory worker. Thus, it appears that TOP $10 \mathrm{~F}^{\prime}$ colonizing the laboratory worker contaminated the material that was eventually PCR amplified into amplicons. These amplicons were further amplified during five rounds of cDNA RDA, possibly increasing the frequency of contaminating DNA fragments. These multiple rounds of PCR have the potential to amplify significant 
amounts of DNA fragments from even a small amount of bacterial contamination in the initial sample. Therefore, to prevent bacterial DNA contamination, extreme caution should be exercised when performing PCR based subtraction techniques, such as cDNA RDA. Prevention of bacterial DNA contamination in such subtractions will enrich the yield of relevant genes, which could result in the identification of new therapeutic targets.

\section{Acknowledgements}

Craig Gawel, Dorothy Guzowski, Colleen Millan and Dr. Robert Pergolizzi of the core facility at NSUH supplied oligonucleotides and performed DNA sequencing. We thank Drs. Nicholas Chiorazzi, James M. Mason, Mamta Naidu, Sangeeta Chavan, Yih-Pai Chu, Mary Berlyn, and Alvin J. Clark for discussion, encouragement, and critical review. This work was supported by the Muriel Fusfeld Foundation, Leonard Wagner Autoimmunity Fund, S.L.E. Foundation, Inc., and the National Institutes of Health (AI44837-01).

\section{References}

1. Klickstein LB. (1998) Production of a subtracted cDNA library. In: Ausubel FM, Brent R, Kingston RE, et al., eds., Current Protocols in Molecular Biology. New York: John Wiley \& Sons, Inc., pp. 5.8.9-5.8.15.

2. Orr SL, Gese E, Hood L. (1992) A new approach to understanding $\mathrm{T}$ cell development: the isolation and characterization of immature CD4-, CD8-, CD3- T cell cDNAs by subtraction cloning. Mol. Biol. Cell 3: 761-773.

3. Zeng J, Gorski RA, Hamer D. (1994) Differential cDNA cloning by enzymatic degrading subtraction (EDS). Nucleic Acids Res. 22: 4381-4385.

4. Yang M, Sytkowski AJ. (1996) Cloning differentially expressed genes by linker capture subtraction. Anal. Biochem. 237: 109-114.

5. Patel M, Sive H. (1998) PCR-based subtractive cDNA cloning. In: Ausubel FM, Brent R, Kingston RE, et al., eds., Current Protocols in Molecular Biology. New York: John Wiley $\delta$ Sons, Inc., pp. 5.9.1-5.9.20.

6. Chu CC, Paul WE. (1998) Expressed genes in interleukin-4 treated B cells identified by cDNA representational difference analysis. Mol. Immunol. 35: 487-502.

7. Argos P, Landy A, Abremski K, et al. (1986) The integrase family of site-specific recombinases: regional similarities and global diversity. ЕMBO J. 5: 433-440.

8. Gopaul DN, Duyne GD. (1999) Structure and mechanism in site-specific recombination. Curr. Opin. Struct. Biol. 9: 14-20.

9. Nunes-Duby SE, Kwon HJ, Tirumalai RS, et al. (1998) Similarities and differences among 105 members of the Int family of site-specific recombinases. Nucleic Acids Res. 26: 391-406.

10. Esposito D, Scocca JJ. (1997) The integrase family of tyrosine recombinases: evolution of a conserved active site domain. Nucleic Acids Res. 25: 3605-3614.

11. Ochman H, Lawrence JG, Groisman EA. (2000) Lateral gene transfer and the nature of bacterial innovation. Nature 405: 299-304.

12. Murakawa Y, Strober W, James SP. (1991) Monoclonal antibody against the human peripheral lymph node homing receptor homologue (Leu 8) inhibits B cell differentiation but not B cell proliferation. J. Immunol. 146: 40-46.

13. Funderud S, Erikstein B, Asheim HC, et al. (1990) Functional properties of CD19+ B lymphocytes positively selected from buffy coats by immunomagnetic separation. Eur. J. Immunol. 20: 201-206.

14. Eisenstein EM, Chua K, Strober W. (1994) B cell differentiation defects in common variable immunodeficiency are ameliorated after stimulation with anti-CD40 antibody and IL-10. J. Immunol. 152: 5957-5968.

15. Altschul SF, Madden TL, Schaffer AA, et al. (1997) Gapped BLAST and PSI-BLAST: a new generation of protein database search programs. Nucleic Acids Res. 25: 3389 3402.

16. Wilson K. (1998) Preparation of genomic DNA from bacteria. In: Ausubel FM, Brent R, Kingston RE, et al., eds., Current Protocols in Molecular Biology. New York: John Wiley \& Sons, Inc., pp. 2.4.1-2.4.5.

17. Kozak M. (1987) An analysis of 5'-noncoding sequences from 699 vertebrate messenger RNAs. Nucleic Acids Res. 15: 81258148.

18. Kozak M. (1991) An analysis of vertebrate mRNA sequences: intimations of translational control. J. Cell Biol. 115: 887-903.

19. Jacob WF, Santer M, Dahlberg AE. (1987) A single base change in the Shine-Dalgarno region of 16S rRNA of Escherichia coli affects translation of many proteins. Proc. Natl. Acad. Sci. USA 84: 4757-4761.

20. Barabino SM, Keller W. (1999) Last but not least: regulated poly(A) tail formation. Cell 99: 9-11.

21. Henkin TM. (2000) Transcription termination control in bacteria. Curr. Opin. Microbiol. 3: 149-153.

22. Nakamura Y, Gojobori T, Ikemura T. (2000) Codon usage tabulated from international DNA sequence databases: status for the year 2000. Nucleic Acids Res. 28: 292.

23. Halling C, Calendar R, Christie GE, et al. (1990) DNA sequence of satellite bacteriophage P4. Nucleic Acids Res. 18: 1649.

24. Sun J, Inouye M, Inouye S. (1991) Association of a retroelement with a P4-like cryptic prophage (retronphage phi R73) integrated into the selenocystyl tRNA gene of Escherichia coli. J. Bacteriol. 173: 4171-4181.

25. Perna NT, Mayhew GF, Posfai G, et al. (1998) Molecular evolution of a pathogenicity island from enterohemorrhagic Escherichia coli O157:H7. Infect. Immun. 66: 3810-3817.

26. Blattner FR, Plunkett G, 3rd, Bloch CA, et al. (1997) The complete genome sequence of Escherichia coli K-12. Science 277: 1453-1474.

27. Moss JE, Cardozo TJ, Zychlinsky A, Groisman EA. (1999) The selC-associated SHI-2 pathogenicity island of Shigella flexneri. Mol. Microbiol. 33: 74-83.

28. Vokes SA, Reeves SA, Torres AG, Payne SM. (1999) The aerobactin iron transport system genes in Shigella flexneri are present within a pathogenicity island. Mol. Microbiol. 33: 63-73.

29. Buchrieser C, Brosch R, Bach S, et al. (1998) The highpathogenicity island of Yersinia pseudotuberculosis can be inserted into any of the three chromosomal asn tRNA genes. Mol. Microbiol. 30: 965-978.

30. Gehring AM, DeMoll E, Fetherston JD, et al. (1998) Iron acquisition in plague: modular logic in enzymatic biogenesis of yersiniabactin by Yersinia pestis. Chem. Biol. 5: 573586.

31. Kovach ME, Shaffer MD, Peterson KM. (1996) A putative integrase gene defines the distal end of a large cluster of ToxR-regulated colonization genes in Vibrio cholerae. Microbiology 142: 2165-2 174 .

32. Sullivan JT, Ronson CW. (1998) Evolution of rhizobia by acquisition of a $500-\mathrm{kb}$ symbiosis island that integrates into a phe-tRNA gene. Proc. Natl. Acad. Sci. USA 95: 51455149.

33. Grant SG, Jessee J, Bloom FR, Hanahan D. (1990) Differential plasmid rescue from transgenic mouse DNAs into Escherichia coli methylation-restriction mutants. Proc. Natl. Acad. Sci. USA 87: 4645-4649.

34. Bachmann BJ. (1996) Derivations and genotypes of some mutant derivatives of Escherichia coli K-12. In: Neidhardt FC 
ed., Escherichia coli and Salmonella: Cellular and Molecular Biology. Washington, D.C.: ASM Press, pp. 2460-2488.

35. Clark AJ, Inwood W, Cloutier T, Dhillon TS. (2001) Nucleotide sequence of coliphage HK620 and the evolution of lambdoid phages. J. Mol. Biol. 311: 657-679.

36. Hickman AB, Waninger S, Scocca JJ, Dyda F. (1997) Molecular organization in site-specific recombination: the catalytic domain of bacteriophage HPl integrase at 2.7 A resolution. Cell 89: 227-237.
37. Kwon HJ, Tirumalai R, Landy A, Ellenberger T. (1997) Flexibility in DNA recombination: structure of the lambda integrase catalytic core. Science 276: 126-131.

38. Guo F, Gopaul DN, van Duyne GD. (1997) Structure of Cre recombinase complexed with DNA in a site-specific recombination synapse. Nature 389: 40-46.

39. Subramanya HS, Arciszewska LK, Baker RA, et al. (1997) Crystal structure of the site-specific recombinase, XerD. EMBO J. 16: 5178-5187. 